\title{
The influence of match outcome on running performance in elite Gaelic football
}

\author{
Shane Mangan \\ Technological University Dublin, shane.mangan@tudublin.ie \\ Shane Malone \\ Technological University Dublin, Shane.Malone@TUDublin.ie \\ Martin Ryan \\ Technological University Dublin
}

See next page for additional authors

Follow this and additional works at: https://arrow.tudublin.ie/ittsciart

Part of the Sports Sciences Commons

\section{Recommended Citation}

Shane Mangan, Shane Malone, Martin Ryan, Jason McGahan, Cian O'neill, Con Burns, Joe Warne, Denise Martin \& Kieran Collins (2017) The influence of match outcome on running performance in elite Gaelic football, Science and Medicine in Football, 1:3, 272-279, DOI: 10.1080/24733938.2017.1363907

This Article is brought to you for free and open access by the School of Science and Computing at ARROW@TU Dublin. It has been accepted for inclusion in Articles by an authorized administrator of ARROW@TU Dublin. For more information, please contact arrow.admin@tudublin.ie, aisling.coyne@tudublin.ie, gerard.connolly@tudublin.ie.

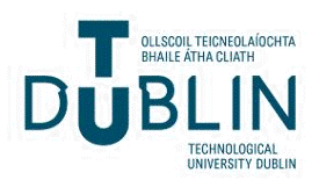




\section{Authors}

Shane Mangan, Shane Malone, Martin Ryan, Jason McGahan, Cian O'Neill, Con Burns, Joe Warne, Denise Martin, and Kieran Collins 


\section{Science and Medicine in Football}

\section{The influence of match outcome on running performance in elite Gaelic football}

\section{Shane Mangan, Shane Malone, Martin Ryan, Jason McGahan, Cian O'neill, Con Burns, Joe Warne, Denise Martin \& Kieran Collins}

To cite this article: Shane Mangan, Shane Malone, Martin Ryan, Jason McGahan, Cian O'neill, Con Burns, Joe Warne, Denise Martin \& Kieran Collins (2017): The influence of match outcome on running performance in elite Gaelic football, Science and Medicine in Football, DOI: 10.1080/24733938.2017.1363907

To link to this article: http://dx.doi.org/10.1080/24733938.2017.1363907

曲 Published online: 14 Aug 2017.

Submit your article to this journal $₫$

Q View related articles $₫$

View Crossmark data $\nearrow$ 


\title{
The influence of match outcome on running performance in elite Gaelic football
}

\author{
Shane Mangan a, Shane Malone, Martin Ryanª, Jason McGahan ${ }^{\mathrm{b}}$, Cian O'neill ${ }^{\mathrm{b}}$, Con Burns ${ }^{\mathrm{b}}$, Joe Warnec, \\ Denise Martin ${ }^{d}$ and Kieran Collins ${ }^{a}$ \\ ${ }^{a}$ Gaelic Sports Research Centre, Institute of Technology Tallaght, Dublin, Ireland; bepartment of Sport, Leisure and Childhood Studies, Cork \\ Institute of Technology, Cork, Ireland; 'Setanta College, Thurles Chamber of Commerce, Thurles, Ireland; dDepartment of Business, Institute of \\ Technology, Blanchardstown, Dublin, Ireland
}

\begin{abstract}
Introduction: Match outcome has been linked to running performance in many sports; however, the association has not yet been examined in Gaelic football. Methods: GPS technologies (4-Hz, VX Sport, Lower Hutt, New Zealand) were used with 5 elite Gaelic football teams over a period of 5 years (20122016), amounting to 95 matches. In total, 780 full match data sets were collected. Game movements were divided into total distance and high-speed distance $\left(\geq 17 \mathrm{~km} \mathrm{~h}^{-1}\right)$. Match outcomes were divided into big losses ( $\geq 6$ points), small losses ( $\leq 5$ points), draws, small wins ( $\leq 5$ points) and big wins ( $\geq 6$ points). Results: Team $(P \leq 0.001)$, playing position $(P \leq 0.001)$ and match outcome $(P \leq 0.034)$ had a significant effect on total and high-speed distance. In the first $(P=0.008)$ and second $(P=0.002)$ quarters of games players ran significantly less high-speed distance in big losses in comparison to draws. In the fourth quarter, players ran significantly less high-speed distance in big losses than in draws $(P=0.001)$, small wins $(P=0.044)$ and big wins $(P=0.011)$. Conclusion: The current study confirms that running performance is a contributing factor to match outcome in Gaelic football. Coaches should be aware how contextual factors can influence running performance.
\end{abstract}

ARTICLE HISTORY

Accepted 25 July 2017

\section{KEYWORDS}

Global positioning systems; match outcome; contextual factors performance analysis

\section{Introduction}

Gaelic football is a field sport native to Ireland. Although an amateur sport, training regimens are similar to professional sports (Beasley 2015). As a consequence of its amateur status, research in the area is quite limited. Gaelic football involves intermittent high-intensity actions, which are influenced by the flow of competitive match-play (Collins et al. 2013). Matches are played between two teams of 15 players over two periods of 35 min (Reilly and Collins 2008). The Gaelic football season consists of two major competitions, which are played over an 8-month period; the national football league and the All-Ireland football championship (Mangan and Collins 2016).

Gaelic football teams play with five positional lines, in addition to a goalkeeper; full back, half back, midfield, half forward and full forward (Malone et al. 2016a). Player role and actual on field position is typically determined by tactics and formation (Carling et al. 2008). Whilst not examined in this sport, research in soccer (Bradley et al. 2011) has observed that different formations will result in different running profiles. Similarly, ball possession has been found to influence the amount of highspeed running that soccer teams will complete (Bradley et al. $2011,2013)$. With this in mind, team differences may exist with relation to running performance in Gaelic football. Player role and position have an influence on the physical demands of players in Gaelic football matches (Collins et al. 2013). The middle three positions (half back, midfield, half forward) cover the highest amount of distance and undertake a greater volume of high-speed running than the other two outfield positions (Malone et al. 2016b). During a match, an elite Gaelic football player will typically cover between $8160 \mathrm{~m}$ and $9222 \mathrm{~m}$, with $1731 \pm 659 \mathrm{~m}$ being at a high speed $\left(\geq 17 \mathrm{~km} \mathrm{~h}^{-1}\right)$ (Malone et al. 2016a, 2016b). Considering Gaelic football matches last only $70 \mathrm{~min}$, relative distances covered in games are similar to that of professional sports such as Australian football (Coutts et al. 2015) and soccer (Barnes et al. 2014).

High-speed running distance and sprint distance have been found to drop in the second half of Gaelic football matches (Malone et al. 2016b). A large percentage decrease in total running performance has been observed between halves for midfielders (11\%), half backs (10.3\%) and half forwards (8.2\%). However, no significant drop off in running performance has been observed for full backs $(0.2 \%)$ or full forwards $(2.5 \%)$ (Malone et al. 2016b). While a drop off in physical performance in the second half of matches could be related to fatigue, it may also be related to the match status (Bradley and Noakes 2013). Soccer players have been observed to cover greater distances at high speed when they are losing than when they are drawing or winning (Lago et al. 2010; Castellano et al. 2011). It's hypothesised that in a losing situation, players increase their work rate in order to draw or win the match (Castellano et al. 2011). A similar trend is evident in Australian football (Sullivan et al. 2014) where players are observed to run $4 \mathrm{~m} \cdot \mathrm{min}^{-1}$ less at high speed when they are winning than when they are losing. Findings relating to running performance and match outcome in rugby league are mixed. One study observed an increase in high-speed running in wins (Gabbett 2013) while another study (Kempton and Coutts 
2015) observed a reduction in high-speed running in wins. Both studies highlight opposition quality as a factor that influences running performance and may explain the difference between the two observations (Gabbett 2013; Kempton and Coutts 2015). The score line effect on work rate may also affect players differently, based on which position they play (Redwood-Brown et al. 2012). Therefore, team differences may influence running performance in Gaelic football, while match outcome may be influenced by running performance.

It is currently unknown whether there is any association between running performance and match outcome in Gaelic football. While global positioning systems (GPS) are useful for monitoring players' external loads, it is unclear whether they can be utilised to provide a link between running metrics and match outcome in Gaelic football. Therefore this paper will examine the influence of total distance $(\mathrm{m})$ and high-speed distance $\left(\geq 17 \mathrm{~km} \mathrm{~h}^{-1} ; \mathrm{m}\right)$ on match outcome in elite Gaelic football. Further to this high-speed distance $\left(\geq 17 \mathrm{~km} \mathrm{~h}^{-1} ; \mathrm{m}\right)$ per quarter will be examined with respect to match outcome. A comparative analysis will also be conducted for team and playing position across all variables. It was hypothesised that match outcome, playing position and team will all have an influence on running performance in Gaelic football. Given the anecdotal evidence that exists, we expected that running performance would be greater in winning matches than in losing matches. We anticipated that half backs, half forwards and midfielders would run greater total distances and highspeed distances than full backs and full forwards.

\section{Methods}

\section{Data collection}

GPS were used to monitor player movements in adult male Gaelic footballers over a period of 5 years (2012-2016). Match data were gathered from 5 elite Gaelic football teams. Participants were selected on the basis that they were chosen to be a part of their respective county squad in that given year and as a result, were seen as the best players in their respective county. The ELO ratings formula for Gaelic football was used to classify the level of quality of each of the teams (Mangan and Collins 2016). The system considers previous results, score margins and opposition quality when determining a team's rating points. As a result, teams can be graded according to their number of points. At the time of data collection, all teams were either classified as Tier 1 teams ( $\geq 1728$ ELO points) or Tier 2 teams (1511-1727 ELO points). No data were collected for Tier 3 or 4 teams. Opposition ranking was not considered for this study. In incidences where a player did not complete the entire match, their results were excluded from analysis. A total of 780 full-match samples, collected from 95 matches, were used for final analysis. Data were collected from the early rounds of the national football league to the later rounds of the All-Ireland football championship. The GPS devices used in the study sample at $4 \mathrm{~Hz}$ (VX Sport, Lower Hutt, New Zealand). The validity and reliability of the selected GPS device has previously been established (Buchheit et al. 2014; Malone et al. 2014). Before going onto the pitch, each player was equipped with a harness for the GPS device. The device was placed within the protective harness between the shoulder blades. Each device was turned on and activated in order to establish a satellite lock for at least 15 min before the start of each match (Maddison and Ni Mhurchu 2009).

Match data were extracted from the GPS devices post game using the VXSport software (VXSport View; Firmware 4.01.2.0). Match files were trimmed to ensure that only the time a player spent on the pitch was recorded. All match data was divided into 4 quarters, each lasting $17.5 \mathrm{~min}$. Once extracted, the data was then exported into a custom spreadsheet (Excel, Microsoft, Redmond, USA). Total distance (m), high-speed distance $\left(\geq 17 \mathrm{~km} \mathrm{~h}^{-1} ; \mathrm{m}\right)$ and minutes spent on the pitch were noted for all players. Total distance was selected as it is a standard measure used in team sports while high-speed distance has previously been reported in Gaelic football (Collins and Doran 2015; Malone et al. 2016a, 2016b). The coefficient of variation (CV) for the selected GPS devices was less than $5 \%$ for total distance, however, highspeed distance had a CV of $8 \pm 2.5 \%$ (95\% Cl) (Malone et al. 2014). Match results were obtained from official match reports. Informed consent and institutional ethical approval were obtained before the commencement of the investigation.

\section{Data analysis}

All running performance data was anonymised before analysis as per institution guidelines. Teams were given a numbered code (1-5) while players were given a positional code (1-15). Playing positions were divided into 5 categories; full back ( $n=189)$, half back $(n=191)$, midfield $(n=108)$, half forward ( $n=166)$ and full forward $(n=126)$. Fulltime scores were noted for each match. In order to calculate the average winning margin, match results $(n=1194)$ for the previous 7 years in the national football league and the All-Ireland football championship were analysed. In matches that resulted in a win for either team, the average score difference was found to be $6.35 \pm 5.54$ points. Using 6 points as the average point difference, wins and losses were split into big ( $\geq 6$ points) and small ( $\leq 5$ points). Score margins have been previously found to influence running performance (Gabbett 2013). Match outcome was categorised into 5 groups; big loss ( $\geq 6$ points: $n=112$ ), small loss ( $\leq 5$ points: $n=172)$, draw $(n=67)$, small win ( $<5$ points: $n=235$ ) and big win ( $\geq 6$ points: $n=194$ ).

\section{Statistical analysis}

Prior to the commencement of statistical analysis, assumptions of normality were assessed. Levene's Test of Equality of Error Variances was failed for both total distance and high-speed distance. Likewise, the data for high-speed distance per quarter failed Box's Test of Equality of Covariance Matrices. This variance could be explained by the fact that positional differences for running performance exist within Gaelic football (Malone et al. 2016b). Statistical analysis was carried out in SPSS for Mac (Statistical Package for the Social Sciences data analysis software V16.0, SPSS Inc., Chicago, Illinois, USA.). Data are presented as means, standard deviation and $95 \%$ Confidence Intervals (Cl). Statistical significance was accepted 
at $a \leq 0.05$. A three-way ANOVA was used to examine the interaction between match outcome, player position and team on total distance $(\mathrm{m})$ and high-speed $\left(\geq 17 \mathrm{~km} \mathrm{~h}^{-1}\right)$ distance (m). A four-way mixed ANOVA with a repeated measure was used to analyse the interaction between match outcome, position and team with the high-speed distance covered across quarters (1-4). Simple main effects were calculated under a Bonferroni correction. Where a significant two-way interaction was observed, a subsequent two-way ANOVA was performed. Tukey post hoc analyses were used to determine within group differences. The partial eta-squared $(\eta 2)$ value was reported as a measure of effect size (Cohen 1988). Effect size benchmarks specific to partial eta-squared (Cohen 1969) were used to divide effects into small (.0099-.0587), medium (.0588-.1378) and large (>.1379) (Richardson 2011).

\section{Results}

\section{Total distance}

There was a statistically significant interaction between team and match outcome on total distance $(P \leq 0.001)$, with a medium effect observed (partial $\eta 2=.068$ ) (Table 1). Team 5 covered a greater amount of distance than all other teams in draws and wins $(P \leq 0.004)$. Similarly, in small losses team 5 ran a greater distance than teams 1,2 and $3(P \leq 0.001)$. In big wins, Team 1 covered a greater amount of distance than team 2 $(P \leq 0.023)$. There were no differences between the other teams.

Position had a large effect on total distance $(P \leq 0.001$, partial $\eta 2=.245$ ). Midfielders covered the greatest distance (9984 $\pm 1805 \mathrm{~m}$ [95\% Cl: $9665-10,302 \mathrm{~m}])$, followed by half forwards (9440 $\pm 1765 \mathrm{~m}$ [95\% Cl: 9183-9697 m]), half backs $(8992 \pm 1582 \mathrm{~m}$ [95\% Cl: 8752-9232 m]), full forwards (7660 $\pm 2009 \mathrm{~m}$ [95\% Cl: 7365-7955 m]) and finally full backs (7418 \pm 1397 m [95\% Cl: 7177-7659 m]) (Figure 1). All positions were significantly different from each other, with the exception of full backs not being significantly different to full forwards. There was no interaction between position and match outcome on total distance $(P=0.696)$, nor was any effect present (partial $\eta 2=0.019$ ).

\section{High-speed distance}

There was a statistically significant interaction between team and match outcome on high-speed running distance $(P \leq 0.001)$, with a medium effect observed (partial $\eta 2=0.060$ ). Team 2 ran less distance at high speed than any other team $(P \leq 0.001)$, while team 5 covered a more distance at high speed than all other teams $(P \leq 0.001)$ (Table 2). A significantly greater $(P \leq 0.017)$ high-speed distance was observed in drawn games for all teams (2342 $\pm 801 \mathrm{~m})$ in comparison to other match outcomes. Players ran less distance at high speed in games that resulted in a big loss in comparison to games that ended in a small win for their team $(P \leq 0.044)$.

Position had a large effect on high-speed distance $(P \leq 0.001$ partial $\eta 2=0.210)$. As with total distance, all positions were significantly different from each other, with the exception of full backs and full forwards (Figure 2). Midfielders covered the greatest amount of high-speed

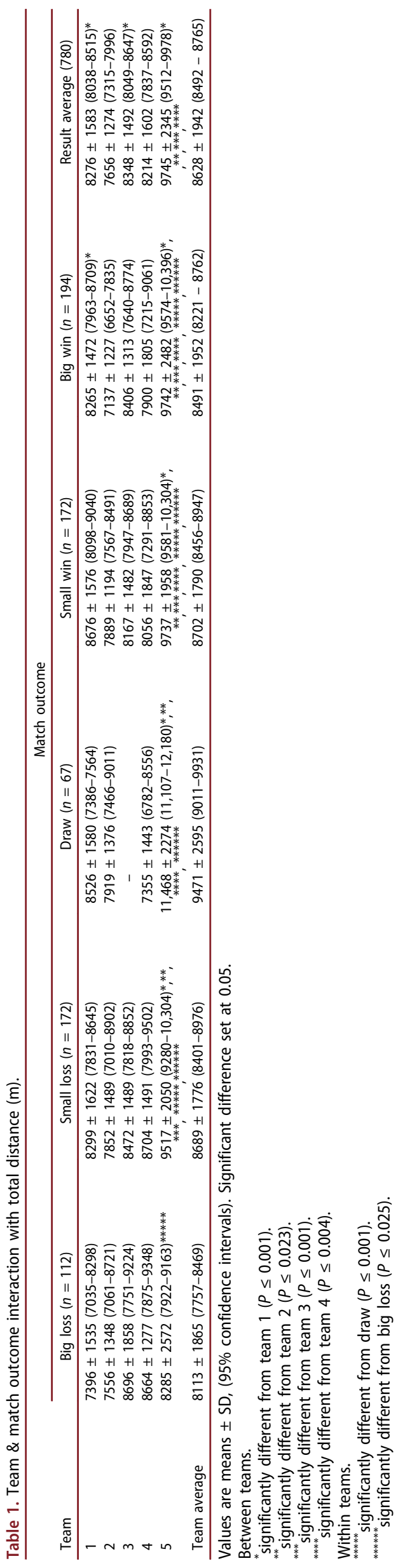




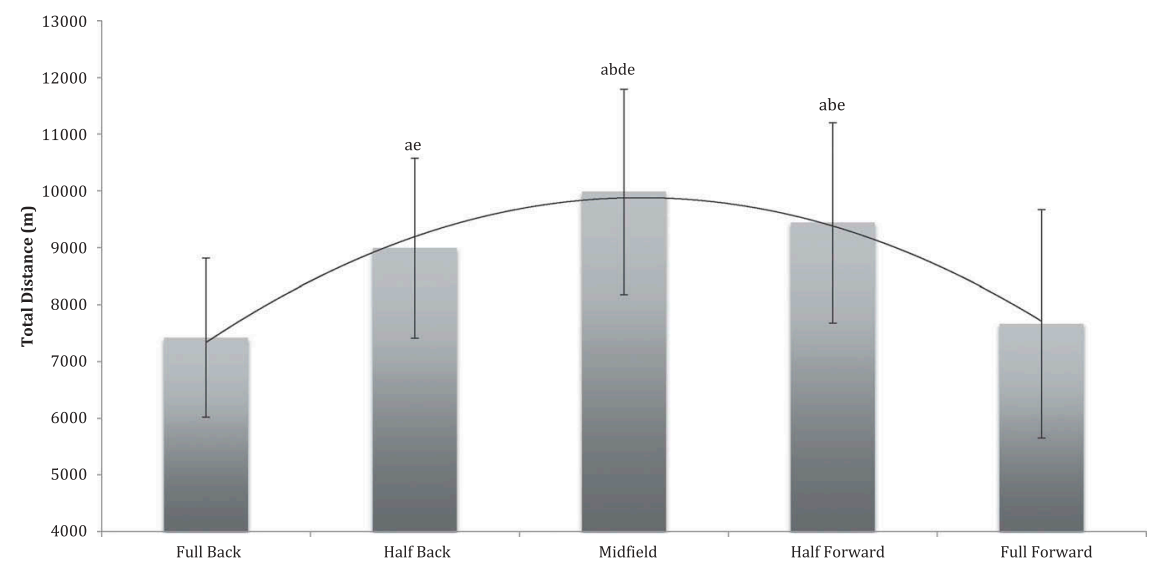

Figure 1. Positional differences for total distance $(m)$ values are means $\pm S D$. Significant difference set at 0.05 .

a - significantly different from full back $(P \leq 0.001)$.

b - significantly different from half back $(P \leq 0.031)$.

$\mathrm{d}$ - significantly different from half forward $(P=0.022)$.

e - significantly different from full forward $(P \leq 0.001)$.

distance (1894 $\pm 623 \mathrm{~m}$ [95\% Cl: 1789-1999 m]), followed by half forwards $(1880 \pm 584 \mathrm{~m}$ [95\% Cl: 1796-1965 m]), half backs $(1724 \pm 583 \mathrm{~m}$ [95\% Cl: 1645-1802 m]), full forwards $(1251 \pm 527 \mathrm{~m}$ [95\% Cl: 1154-1348 m]) and last full backs (1204 $\pm 470 \mathrm{~m}$ [95\% Cl: 1124-1283 m]). There was no interaction between position and match outcome on high-speed distance $(P=0.561$, partial $\eta 2=0.021)$.

\section{Quarter difference for high-speed distance}

A small effect was evident between quarters for high-speed distance $(P \leq 0.001$, partial $\eta 2=.049)$. It was evident that highspeed distance was reduced in quarters two, three and four in comparison to the first quarter $(P \leq 0.001)$. High-speed distance in the fourth quarter was significantly lower than in all other quarters $(P \leq 0.001)$. Position had a small effect on highspeed distance per quarter $(P \leq 0.001$, partial $\eta 2=.014)$. Smallto-medium decreases in high-speed distance across quarters were observed for half backs $(P \leq 0.001$, partial $\eta 2=.101)$, midfielders $(P \leq 0.001$, partial $\eta 2=.097)$, half forwards $(P \leq 0.001$, partial $\eta 2=.131)$ and full forwards $(P \leq 0.001$, partial $\eta 2=.052$ ). There were significant large effects between positions for Quarter $1(P \leq 0.001$, partial $\eta 2=.232)$, Quarter 2 $(P \leq 0.001$, partial $\eta 2=.178)$, Quarter $3(P \leq 0.001$, partial $\eta 2=.175)$ and Quarter $4(P \leq 0.001$, partial $\eta 2=.133)$. Half backs, midfielders and half forwards ran significantly greater high-speed distances than full backs and full forwards in each of the four quarters $(P \leq 0.001)$.

There was a statistically significant interaction between team and match outcome on high-speed distance run per quarter, yet the effect size was small $(P \leq 0.001$, partial $\eta 2=0.041)$. Large effects were observed for match outcome in Quarter $1(P \leq 0.001$, partial $\eta 2=.822)$ and Quarter 4 $(P \leq 0.001$, partial $\eta 2=.811)$ while a small effect was observed in Quarter $2(P=0.005$, partial $\eta 2=.019)$. No effect was observed between match outcomes for high-speed distance in Quarter $3(P=0.307$, partial $\eta 2=.006)$. Post hoc analysis revealed that in Quarter 1 players covered a considerably lower distance at high-speed in big losses in comparison to draws $(P=0.008)$. In Quarter 2, a similar difference was noted between big losses and draws $(P=0.002)$ and between big wins and draws $(P=0.024)$. In Quarter 4 , players ran significantly less in big losses than in draws $(P=0.001)$, small wins $(P=0.044)$ and big wins $(P=0.011)$.

Medium to large effects were evident between teams for all quarters - Quarter $1(P \leq 0.001$, partial $\eta 2=.126)$, Quarter 2 $(P \leq 0.001$, partial $\eta 2=.178)$, Quarter $3(P \leq 0.001$, partial $\eta 2=.128)$ and Quarter $4(P \leq 0.001$, partial $\eta 2=.123)$. Team 5 covered greater distance at high-speed than all other teams in each of the four quarters $(P \leq 0.001)$. On the other end of the scale, team 2 covered less distance at high-speed than all teams in Quarter $1(P \leq 0.001)$, Quarter $2(P \leq 0.045)$ and Quarter $3(P \leq 0.003)$. Additionally, in Quarter 4 , team 2 was observed to cover significantly less distance at high speed than teams $1(P=0.015), 4(P=0.005)$ and $5(P \leq 0.001)$.

\section{Discussion}

The current investigation is the most comprehensive study to date relating to match running demands in elite Gaelic football. This study is the first to investigate the relationship between running performance and match outcome in Gaelic football. The main finding is that team, match outcome and playing position all have small to large effects on total distance and high-speed distance covered by elite Gaelic football players. Players were observed to cover greater distances in games that ended in draws or narrow score margins. During the fourth quarter of matches, teams who lost by a big margin ( $\geq 6$ points) ran significantly less high-speed distance than teams that had drawn or won. Likewise, in the first quarter players covered a lower volume of high-speed distance in big losses than in other results, suggesting a high work-rate in the first quarter is essential. In addition, there appears to be large team specific differences, with teams 2 and 5 significantly different to all other teams for high-speed distance.

The figures for total distance are in line with previous research in elite Gaelic football (Malone et al. 2016a, 2016b). 


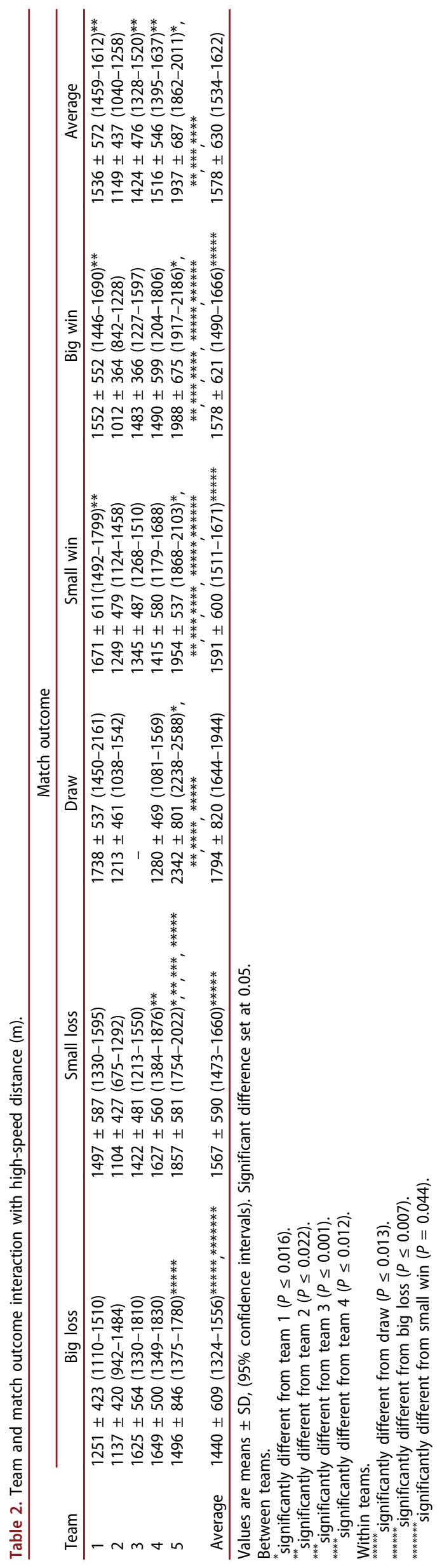

However, the average distance covered at a high speed is lower than previously observed (Malone et al. 2016b). This difference may be explained by the wider range in quality of the teams in this research (Mangan and Collins 2016). In rugby league, lowerranked teams are observed to cover a greater distance at high speed than higher-ranked teams (Gabbett 2013). Positional differences were noted for both total distance (Figure 1) and high-speed distance (Figure 2). These findings are in line with our hypothesis and previous research in Gaelic football where midfielders, half backs and half forwards were found to cover significantly greater distances than full backs and full forwards (Collins et al. 2013; Malone et al. 2016b).

Our data show that there is an association between running performance and match outcome in Gaelic football matches. It remains to be seen whether the distance covered influences the score line or vice versa. The relationship between both is most likely mutual. With the highest distances being covered in drawn games it may indicate the closer a game is, the more a player will run. Caution must be used however as the sample size of drawn games was much lower than other outcomes. An examination of big and small wins and losses suggests that the previous statement holds merit, regardless of the sample size of drawn games. On average, players covered a greater distance in winning matches than in losing matches, a trend observed previously in rugby league (Gabbett 2013). However, in Australian football (Sullivan et al. 2014) and soccer (Lago et al. 2010; Castellano et al. 2011), players are observed to run less in winning matches than in losing matches, contradicting our findings. The lack of agreement in the relationship between running performance and match outcome in these sports results could be explained by the different game structures in Australian football, rugby league and soccer (Varley et al. 2014). We observed no considerable difference between teams for big losses, with teams running a similar amount of distance. This suggests that there could be a minimum work rate that teams need to reach in order to avoid a big loss. Unlike in soccer (Andrzejewski et al. 2016), an interaction between position and match outcome on running distance was not evident.

Differences for total distance (Table 1) and high-speed distance (Table 2) were observed across the individual teams analysed in this study. These findings suggest that style of play or team quality can influence running distance during Gaelic football match play. The difference between teams is most pronounced when comparing teams 2 and 5. Team 2 covered a significantly lower distance at high speed than any other team $(P \leq 0.001)$ while team 5 covered a significantly greater distance at high speed than any other team $(P \leq 0.001)$. No major differences in rating points existed between both teams in any of the seasons examined, suggesting that style of play may cause the difference in running performance. Future research should examine the relationship between team style and physical performance.

Previous research has shown that players will run considerably less in quarters two, three and four $(P \leq 0.001)$ than they will in the first quarter, regardless of result (Malone et al. 2017). It is evident from our findings that in each of the four quarters, players will run less distance at high speed in big losses than in matches with a different outcome. A substantial difference between big losses and draws is evident even in the first quarter (Figure 3). This suggests that a poor work rate in the first quarter may be detrimental to a team's chances of being competitive in a 


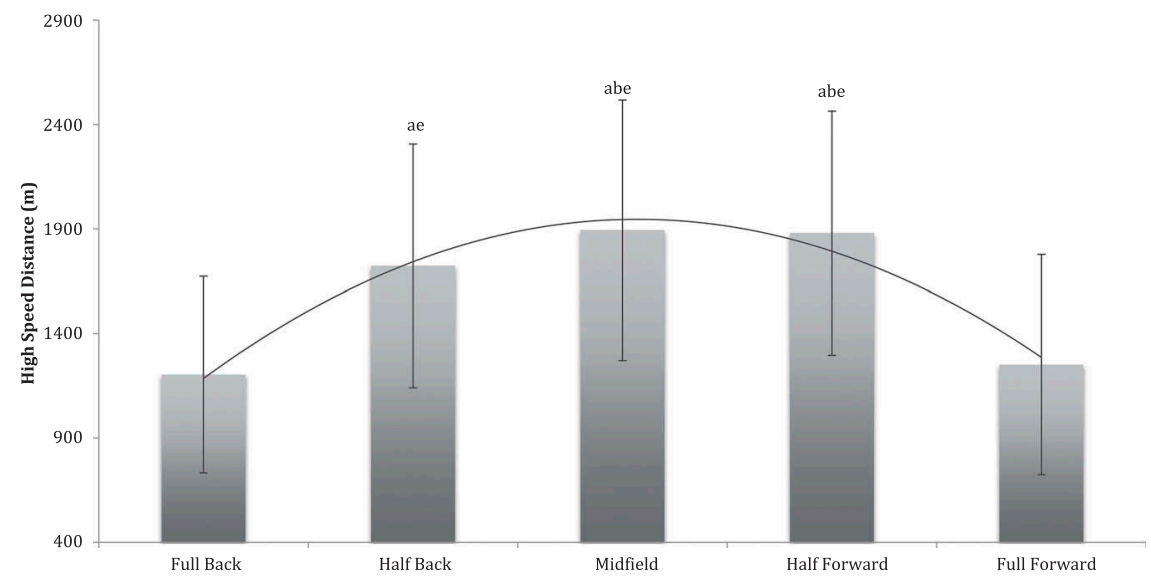

Figure 2. Positional differences for high-speed distance $(\mathrm{m})$ values are means \pm SD. significant difference set at 0.05 .

a - significantly different from full back $(P \leq 0.001)$.

$\mathrm{b}$ - significantly different from half back $(P \leq 0.024)$.

e - significantly different from full forward $(P \leq 0.001)$.

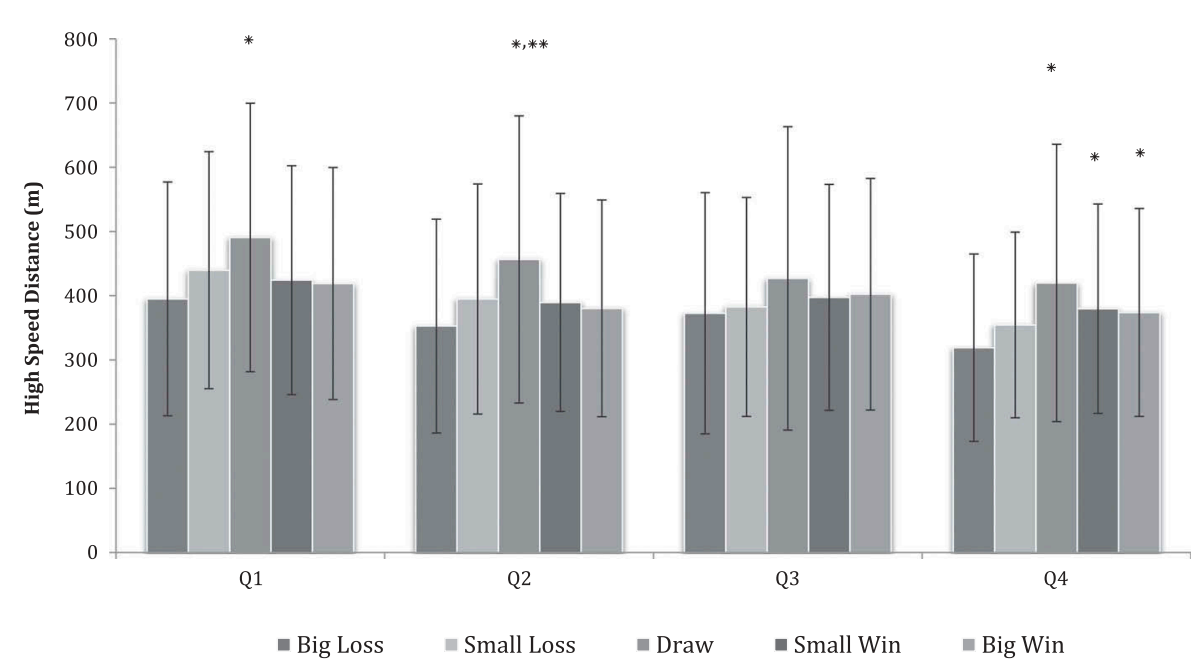

Figure 3. Match outcome differences per quarter for high-speed distance $(\mathrm{m})$ values are means \pm SD. Significant difference set at 0.05 . A significant difference was evident between quarters for high-speed distance $(P \leq 0.001)$. High-speed distance is significantly higher in quarter one than all other quarters $(P \leq 0.001)$. Highspeed distance is significantly lower in quarter four than to all other quarters $(P \leq 0.001)$.

*Significantly different from big loss $(P \leq 0.044)$.

**Significantly different from big win $(P=0.024)$.

match. In the second quarter, players run less in big losses and big wins than in draws. The difference between big wins and draws suggests that pacing may already be a factor in the second quarter. It's been previously suggested that player's subconscious decisions influence their movement within a match in attempt to save energy (Coutts et al. 2010). In the final quarter, players are observed to run significantly less distance at high speed in big losses in comparison to draws and wins. It raises the question whether teams perform less high-speed actions when they are defending as opposed to attacking, as is the case in soccer (Bradley et al. 2013). The difference between match outcomes in the fourth quarter could have a number of explanations. The first being that in big losses, the team's perception of effort exceeds the potential reward (of winning) and motivation is reduced (Paul et al. 2015). Another possible explanation is that certain players/teams are conditioned better than others.

Caution must be used when comparing the results of this study to other Gaelic football teams since anecdotal evidence suggests that different sampling rates of GPS devices can influence results. To calculate the high-speed distance, distance run was broken into different speed zones. A limitation of this method is that the speed zones were not individualised for each player. An approach basing speed zones on physiological thresholds would perhaps be a better reflection on within player and between player differences (Lovell and Abt 2013). A further limitation is that no tactical information or technical performance indicators were examined in association with the running performance data. This limited the conclusions that could be drawn from the inter team differences. Match location (Castellano et al. 2011) and weather (Watanabe et al. 2016) are both factors that have been found to influence running performance however neither were examined in this study. Data in this study were collected from both the national football league and the All-Ireland football championship. It has been suggested that match demands are higher in the All-Ireland football championship (Mangan and Collins 2016) however this difference was not examined in the current study. The findings of 
the current research suggest that closer games require a greater physical exertion. Future research should examine the relationship between score difference and running performance, irrespective of result. In addition to the three factors examined in this study, many other factors including score line, playing style and team quality may also influence work rate within matches. Further investigations are needed in Gaelic football relating to contextual factors and running performance.

\section{Conclusion}

The results of this study confirm that running performance impacts match outcome. Total distance and high-speed distance have a medium effect on match outcome. Coaches should take this into account in their match analyses and when examining match-match variation. When the final score is close, the running demands of the game are increased. The current finding gives coaches another consideration when deciding whether to make substitutions, with score line possibly influencing the rate of fatigue. Teams must train in anticipation for a worst-case scenario, whereby the match demands are increased due to the match status. These findings may promote reductions in weekly training loads in the buildup to a match, where a close game is expected, or in the week following a close match. Playing position was found to have a large effect on running performance. Coaches should ensure training games are competitive in terms of score line in order to replicate the running performance observed in close games and also individualised based on playing position. Team differences are evident for running distance in matches, suggesting that tactics and style of play have a major influence on performance. With this in mind, different opposition may also have an influence on running demands. In the final quarter of games players run less when they lose by big margins than when they draw or win. This finding may influence game strategies in the final quarter of matches. Match analyses should not take running performance in isolation, however, since tactics and technical performance also play an important role in influencing match outcome. By understanding the factors that influence running performance in matches, teams can adopt their match preparation strategies accordingly.

\section{Practical Implications}

This is the first study to show that there is a relationship between running performance and match outcome in Gaelic football. Coaches should increase recovery time for players following close games. The results also show that team differences exist with respect to running performance. Coaches should be aware that their teams style of play and tactics may influence their teams running performance. One must be aware that many more contextual factors may influence running performance in addition to the four examined in this research (match quarter, playing position, match outcome and team).

\section{Disclosure statement}

No potential conflict of interest was reported by the authors.

\section{References}

Andrzejewski M, Konefał M, Chmura P, Kowalczuk E, Chmura J. 2016. Match outcome and distances covered at various speeds in match play by elite German soccer players. Int J Perform Anal Sport. 16:817-828.

Barnes C, Archer DT, Hogg B, Bush M, Bradley PS. 2014. The evolution of physical and technical performance parameters in the English Premier League. Int J Sports Med. 35:1095-1100.

Beasley KJ. 2015. Nutrition and Gaelic football: review, recommendations, and future considerations. Int J Sport Nutr Exerc Metab. 25:1-13.

Bradley PS, Carling C, Archer D, Roberts J, Dodds A, Di Mascio M, Paul D, Diaz AG, Peart D, Krustrup P. 2011. The effect of playing formation on high-intensity running and technical profiles in English FA Premier League soccer matches. J Sports Sci. 29:821-830.

Bradley PS, Lago-Peñas C, Rey E, Gomez Diaz A. 2013. The effect of high and low percentage ball possession on physical and technical profiles in English FA Premier League soccer matches. J Sports Sci [Internet]. 31:1261-1270. http://www.ncbi.nlm.nih.gov/pubmed/23697463.

Bradley PS, Noakes TD 2013. Match running performance fluctuations in elite soccer: indicative of fatigue, pacing or situational influences? J Sports Sci [Internet]. 31:1627-1638. http://www.ncbi.nlm.nih.gov/ pubmed $/ 23808376$

Buchheit M, Allen A, Poon TK, Modonutti M, Gregson W, Di Salvo V. 2014. Integrating different tracking systems in football: multiple camera semiautomatic system, local position measurement and GPS technologies. J Sports Sci [Internet]. 32:1844-1857. http://search.ebscohost.com/login. aspx?direct=true $\& \mathrm{db}=\mathrm{s} 3 \mathrm{~h} \& \mathrm{AN}=99907346 \&$ site $=$ ehost-live.

Carling C, Bloomfield J, Nelsen L, Reilly T. 2008. The role of motion analysis in elite soccer: contemporary performance measurement techniques and work rate data. Sport Med. 38:839-862.

Castellano J, Blanco-Villaseñor A, Álvarez D. 2011. Contextual variables and time-motion analysis in soccer. Int J Sports Med. 32:415-421.

Cohen J. 1969. Statistical power analysis for the behavioural sciences. New York (NY): Academic Press.

Cohen J. 1988. Statistical power analysis for the behavioral sciences. Stat Power Anal Behav Sci. 2nd:567.

Collins DK, Doran D. 2015. The performance profile and physical demands of elite Gaelic football. In: Bangsbo J, Krustrup P, editors. Presented at the 8th World Congress on Science and Football.

Collins K, Solan B, Doran DA. 2013. A preliminary investigation into highintensity activity during elite Gaelic football. J Sport Ther. 1:10.

Coutts AJ, Kempton T, Sullivan C, Bilsborough J, Cordy J, Rampinini E. 2015. Metabolic power and energetic costs of professional Australian Football match-play. J Sci Med Sport [Internet]. 18:219-224. [Accessed 2016 Dec 19]. http://www.ncbi.nlm.nih.gov/pubmed/24589369.

Coutts AJ, Quinn J, Hocking J, Castagna C, Rampinini E. 2010. Match running performance in elite Australian Rules Football. J Sci Med Sport. 13:543-548.

Gabbett T. 2013. Influence of the opposing team on the physical demands of elite rugby league match play. J Strength Cond Res [Internet]. 27:1629-1635. http://www.ncbi.nlm.nih.gov/pubmed/23037616.

Kempton T, Coutts AJ. 2015. Factors affecting exercise intensity in professional rugby league match-play. J Sci Med Sport [Internet]. 19:504-508. http://www.ncbi.nlm.nih.gov/pubmed/26117160.

Lago C, Casais L, Dominguez E, Sampaio J. 2010. The effects of situational variables on distance covered at various speeds in elite soccer. Eur $J$ Sport Sci [Internet]. 10:103-109. [Accessed 2016 Nov 14]. http://www. tandfonline.com/doi/abs/10.1080/17461390903273994.

Lovell R, Abt G. 2013. Individualization of time-motion analysis: a casecohort example. Int J Sports Physiol Perform. 8:456-458.

Maddison R, Ni Mhurchu C. 2009. Global positioning system: a new opportunity in physical activity measurement. Int J Behav Nutr Phys Act [Internet]. 6:73. http://www.pubmedcentral.nih.gov/articlerender. fcgi?artid=2777117\&tool=pmcentrez\&rendertype=abstract.

Malone S, Doran D, Collins K, Morton J, McRobert A 2014. Accuracy and reliability of VXsport global positioning system in intermittent activity. In: Amsterdam: Proceedings of the 19th Annual Congress of the European College of Sport Science.

Malone S, Solan B, Collins K. 2017. The running performance profile of elite Gaelic football match-play. J Strength Cond Res. 31:1-25. 
Malone S, Solan B, Collins K, Doran D. 2016a. The metabolic power and energetic demands of elite Gaelic football match play. J Sport Medince Phys Fit. 57:1-20.

Malone S, Solan B, Collins K, Doran D. 2016b. Positional match running performance of elite Gaelic football. J Strength Cond Res [Internet]. 30:2292-2298. http://content.wkhealth.com/linkback/openurl?sid= WKPTLP:landingpage\&an $=00124278-900000000-96629$.

Mangan S, Collins K. 2016. A rating system for Gaelic football teams: factors that influence success. Int J Comput Sci Sport [Internet]. 15:78-90. http://iacss.org/index.php?id=30.

Paul D, Bradley P, Nassis G. 2015. Factors affecting match running performance of elite soccer players: shedding some light on the complexity. Int J Sports Physiol Perform [Internet]. 10:516-519. http://www.ncbi. nlm.nih.gov/pubmed/25928752.

Redwood-Brown A, Donoghue PO, Robinson G, Neilson P. 2012. The effect of score-line on work-rate in English FA Premier League soccer. Int J Perform Anal Sport. 12:258-271.
Reilly T, Collins K. 2008. Science and the Gaelic sports: Gaelic football and hurling. Eur J Sport Sci [Internet]. 8:231-240. [Accessed 2016 Dec 18]. http://www.tandfonline.com/doi/abs/10.1080/ 17461390802251851.

Richardson J. 2011. Eta squared and partial eta squared as measures of effect size in educational research. Educ Res Rev. 6:135-147.

Sullivan C, Bilsborough JC, Cianciosi M, Hocking J, Cordy J, Coutts AJ. 2014. Match score affects activity profile and skill performance in professional Australian Football players. J Sci Med Sport [Internet]. 17:326-331.

Varley MC, Gabbett T, Aughey RJ. 2014. Activity profiles of professional soccer, rugby league and Australian football match play. J Sports Sci [Internet]. 32:1858-1866. [Accessed 2017 Mar 6]. http://www.ncbi.nlm. nih.gov/pubmed/24016304.

Watanabe N, Wicker P, Yan G. 2016. Weather conditions, travel distance, rest, and running performance: the 2014 FIFA World Cup and Implications for the Future. J Sport Manag. 31:1-37. 